\title{
A YARN-BASED BACTERIA-POWERED BATTERY FOR SMART TEXTILES
}

\author{
Yang Gao, Lin Liu, and Seokheun Choi*,
}

Bioelectronics \& Microsystems Laboratory, Department of Electrical \& Computer Engineering, Center for Research in Advanced Sensing Technologies \& Environmental Sustainability (CREATES), State University of New York at Binghamton, New York, USA

\begin{abstract}
We report a flexible and wearable bacteria-powered battery in which four functional yarns are placed in parallel for biological energy harvesting. A current collecting yarn is sandwiched between two conductive/hydrophilic active yarns including electricity-generating bacteria while a polymer-passivated cathodic yarn is located next to one of the active yarns to form a biological fuel cell configuration. The device uses Shewanella oneidensis MR-1 as a biocatalyst to produce a maximum power of $17 \mu \mathrm{W} / \mathrm{cm}^{3}$ and current density $327 \mu \mathrm{A} / \mathrm{cm}^{3}$, which are enough to power small-power applications. This yarn-structured biobattery can be potentially woven or knitted into an energy storage fabric to provide a higher power for smart textiles. Furthermore, sweat generated from the human body can be a potential fuel to support bacterial viability, providing the long-term operation of the battery.
\end{abstract}

\section{INTRODUCTION}

Textile-based wearable electronics have recently emerged as a technology that promises next generation, ubiquitous health monitoring $[1,2]$. Even under extreme mechanical deformation, their flexible and stretchable characteristics allow conformal and reliable contact with asymmetrical and non-uniform human skin with minimum discomfort. However, there has been a significant challenge in creating a truly self-reliant and stand-alone wearable sensing system that does not rely on an external power source [3]. Recently, our group, for the first time, demonstrated a textilebased bacteria-powered biobattery and ensured its practical efficacy as a flexible and stretchable power supply [4, 5]. Bacteria-powered biobatteries are arguably the most underdeveloped for wearable electronic applications because microbial cytotoxicity may pose health concerns. However, if we consider that humans possess many bacterial cells more than human cells in their bodies, the direct use of bacterial cells as a power resource interdependently with the human body is conceivable for wearable electronics. Our previous research efforts in flexible bacteria-powered batteries focused on integrating electronic components into a single sheet of 2-D fabric through a top-down approach $[4,5]$.

In this work, we presented a yarn-based biobattery for flexible and wearable textile integration (Figure 1). The realization of biobattery on flexible substrates, such as paper or textile, has been mentioned in literature [4-6]. Unlike the prior arts where functional regions and parts are constructed directly on the substrate, direct functionalization of basic yarns can enable more flexible and controllable device fabrication in a smaller device footprint, enhance the versatility of the device design and improve performance per unit volume. This bacteria-powered energy harvester was built on 1-D yarns through a bottom-up approach from which various electronic components of a large textile can be weaved or knitted.

\section{MATERIALS AND EXPERIMENTAL SET-UP Device Fabrication}

The anodic compartment consisted of three yarns; a conductive thread as a current collector and two PEDOT:PSS

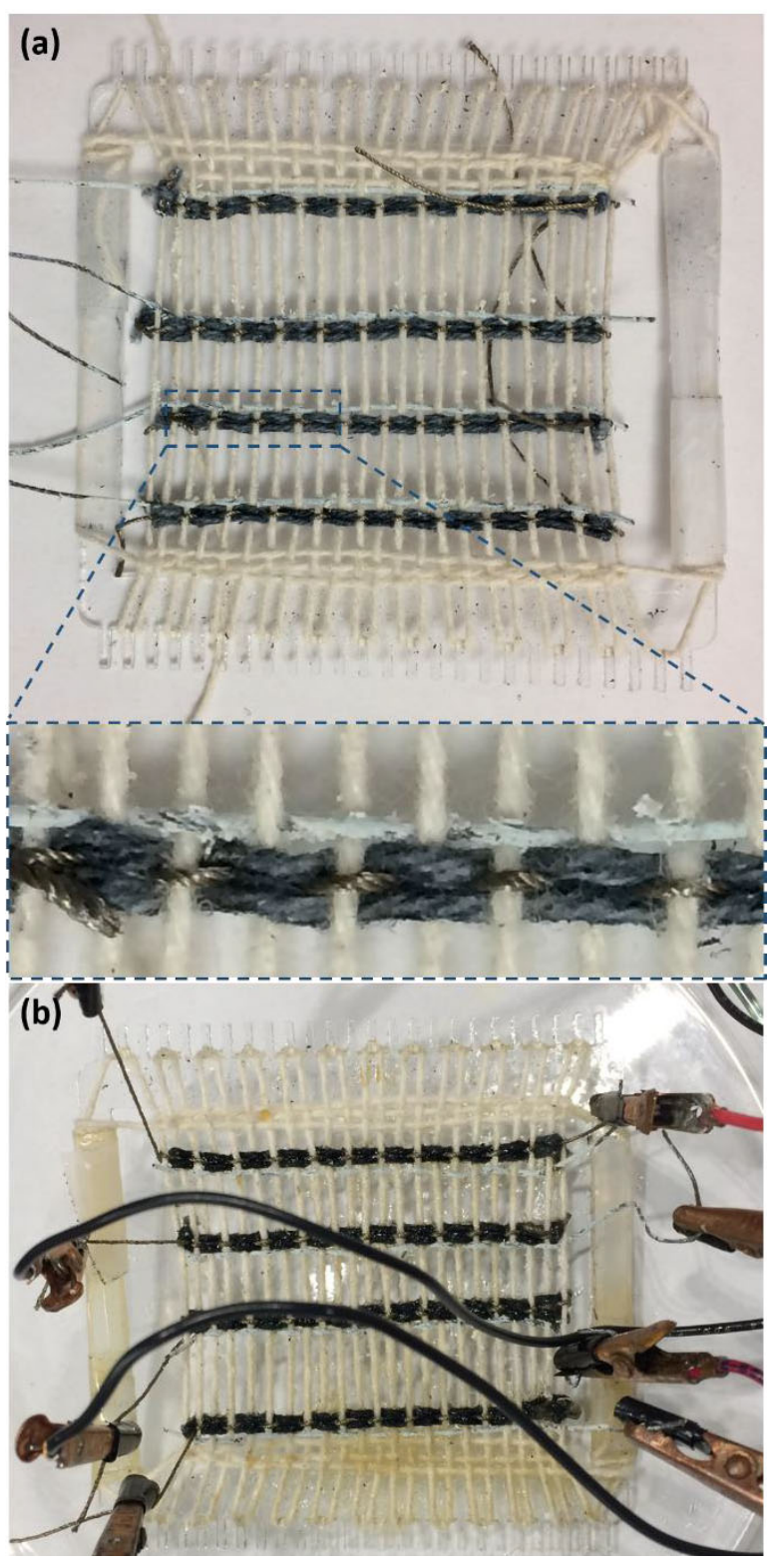

Figure 1. (a) Photo-images showing the textile weaving process of four yarn-based biobatteries and the enlarged view of the interlaced structure of one biobattery with four functional yarns. (b) Operating biobatteries.

(poly(3,4-ethylenedioxythiophene) polystyrene sulfonate) functionalized cotton yarns (or active yarns) were placed in parallel shown in (Figure $1 \&$ Figure 2). The cathodic yarn was based on the same conductive thread as the anode. The silverbased cathodic paste was first applied the cathodic yarn and a microporous polytetrafluoroethylene (PTFE) membrane as a proton exchange membrane was coated over the cathode yarn. 


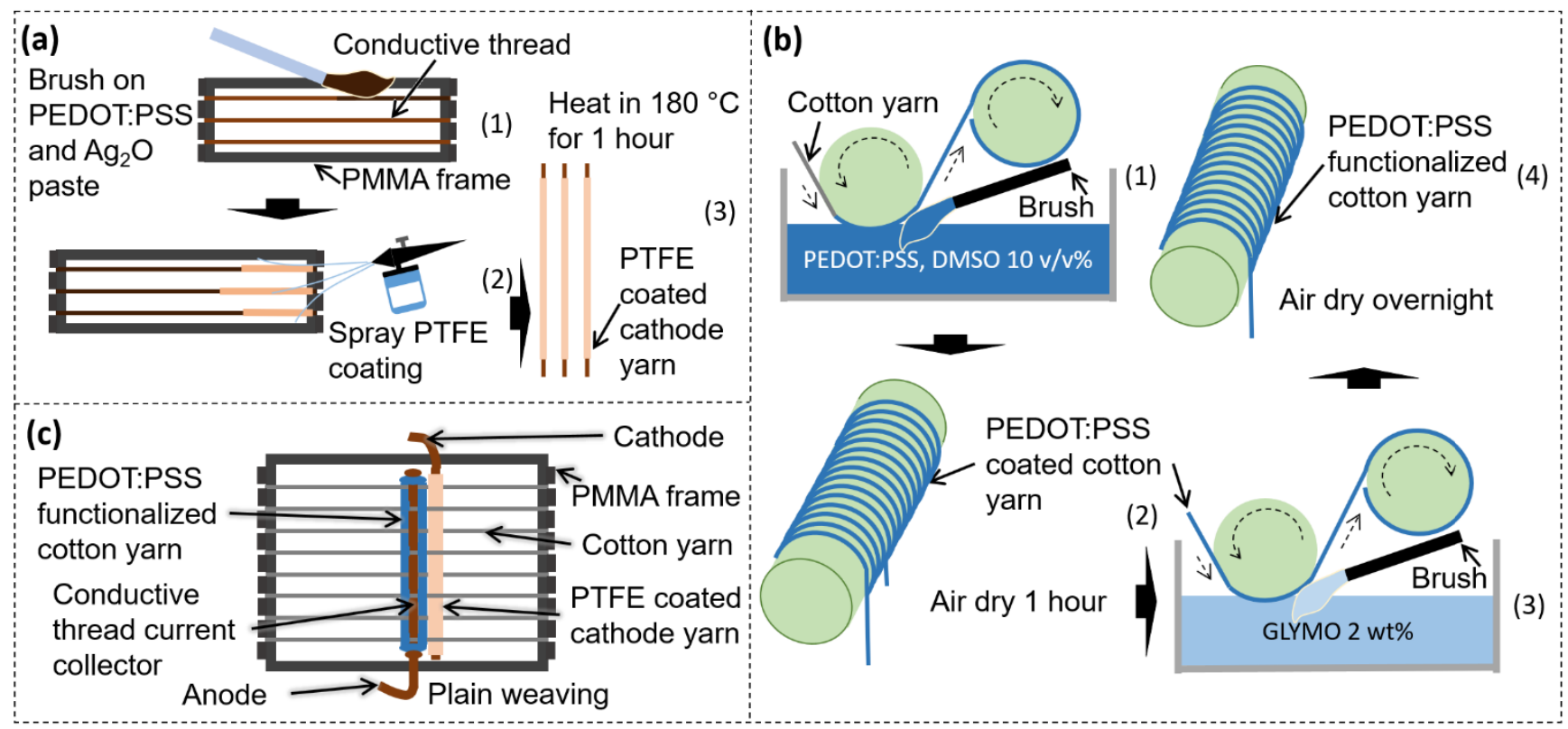

Figure 2: Schematic illustration of the processes employed to fabricate yarn-based biobattery, (a) cathodic yarns, (b) anodic yarns and (c) woven textile with anode and cathode in weft direction.

Four functional yarns were weaved into a plain weaving pattern (Figure 2) as the prototype yarn-based biobattery (Figure 3).

\section{Cathodic yarn fabrication}

Commercial cotton yarns were first sterilized by immersing in $70 \mathrm{v} / \mathrm{v} \%$ ethanol with sonication for $30 \mathrm{~min}$, followed by drying in a ventilated oven at $50^{\circ} \mathrm{C}$. The silver-based cathodic paste was prepared by mixing silver (I) oxide (Sigma-Aldrich) with $1 \mathrm{wt} \%$ PEDOT:PSS. The PEDOT:PSS weight percentage increased from $1 \mathrm{wt} \%$ to $2 \mathrm{wt} \%$ by further heating and stirring processes on a hot plate stirrer $\left(60^{\circ} \mathrm{C}\right.$ with $\left.110 \mathrm{rpm}\right)$. After the pre-cleaned conductive threads were stretched on the poly(methyl methacrylate) (PMMA) frame, the cathodic paste was brushed onto the thread and airdried (Figure 2a). To passivate the treated cathodic yarn, $60 \mathrm{wt} \%$ PTFE water emulsion was mixed with isopropyl alcohol and sonicated for $30 \mathrm{~min}$, yielding a uniform $40 \mathrm{wt} \%$ PTFE emulsion. The emulsion was sprayed onto the conductive cathodic thread and dried at $180^{\circ} \mathrm{C}$ for $1 \mathrm{~h}$.

\section{Anodic yarn fabrication}

Conductive ink was prepared by mixing $1 \mathrm{wt} \%$ PEDOT:PSS (Clevios ${ }^{\mathrm{TM}} \mathrm{PH} 1000$, Heraeus) with dimethyl sulfoxide (DMSO, Sigma-Aldrich) at a volume ratio of 9:1. The cleaned cotton yarns were pulled through PEDOT:PSS conductive ink via a customized reeling setup (Figure 2b). A brush was pressed on the cotton yarns to facilitate the conductive ink penetration. The conductive cotton yarns were air-dried for $1 \mathrm{~h}$ to allow the solvent to evaporate. Then, the yarns were treated with $2 \mathrm{wt} \%$ 3-glycidoxypropyltrimethoxysilane (GLYMO, Sigma-Aldrich) to improve its hydrophilic property. The functionalized yarns were further airdried overnight.

\section{Weaving of biobatteries}

The cleaned non-conductive threads were used as warp yarns and held stationary in the PMMA frame (Figure 2c). The anodic

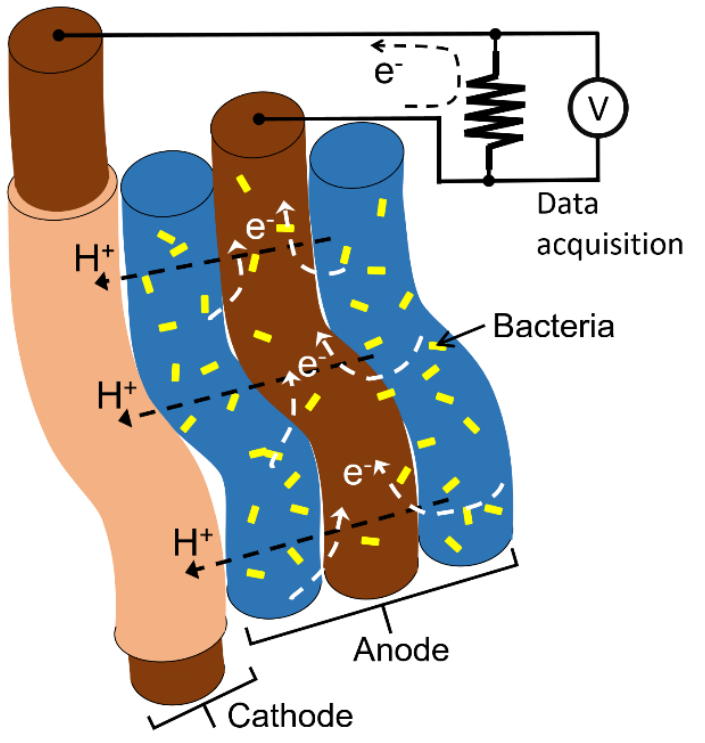

Figure 3: Schematic diagram of the yarn-based biobattery.

and cathodic yarns were weaved through the warps in a plain pattern (Figure 3).

\section{Inoculum}

Cryostock Shewanella oneidensis MR-1 was cultured in $3 \mathrm{wt} \%$ soy broth media for $24 \mathrm{~h}$, and diluted with fresh media. Growth was monitored by measuring the optical density at $600 \mathrm{~nm}\left(\mathrm{OD}_{600}\right)$, and the culture reached an $\mathrm{OD}_{600}$ of 1.6.

\section{Operating principle and measurement setup}

The yarn-based biobatteries were connected to a National Instrument (NI) data acquisition module (USB-6212) with a customized user interface for voltage measurements. To activate the biobattery, $260 \mu \mathrm{L}$ of bacterial inoculum was pipetted to each 

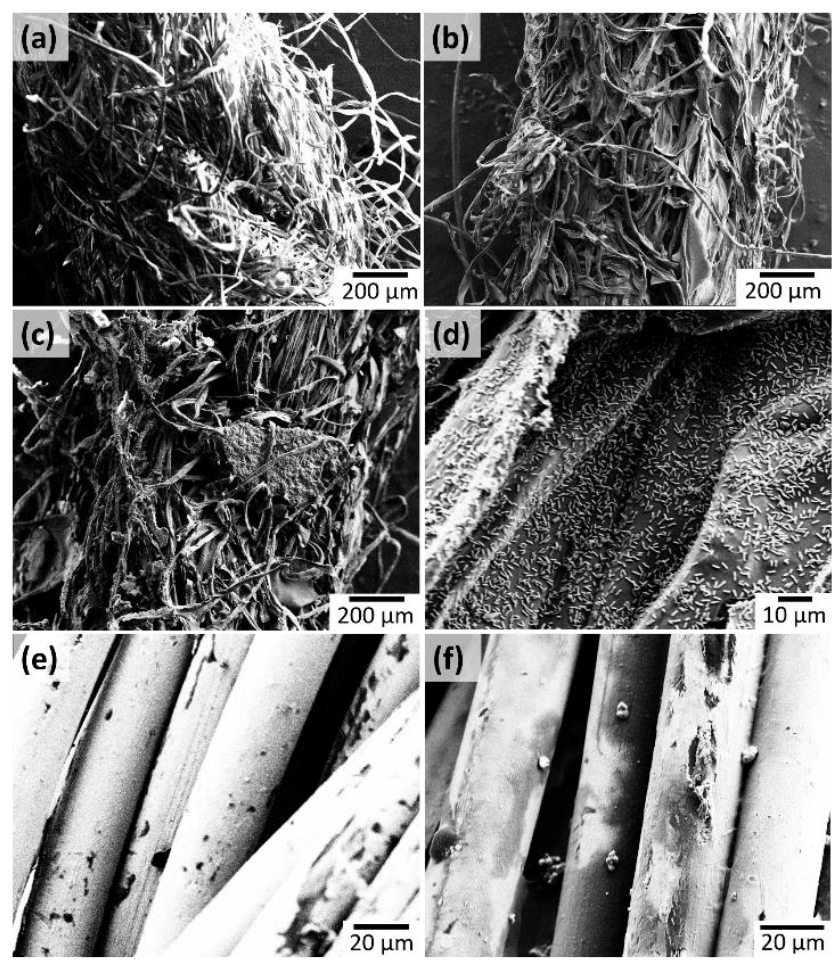

Figure 4: SEM images of (a) the cotton yarn without treatment, (b) the PEDOT:PSS functionalized anodic cotton yarn, $(c \& d)$ the anodic yarns after inoculation, (e) the conductive cathodic yarn and (f) the cathodic yarn with PTFE coating.

device. Resistive loads were connected to the biobatteries, and the output current and power were calculated via Ohm's law.

\section{RESULTS AND DISCUSSION}

The cotton yarn (Figure 4a) was chosen because of its good hydrophilicity, resistance to organic solvents, fabric integrity at elevated temperatures and low cost [7]. Adding the conductive polymer PEDOT:PSS to the cotton yarn created a conductive matrix with the high specific surface, improving bacterial attachment and their respiration (Figure 4b, 4c \& 4d) [5, 8]. Therefore, the treatment for the conductive yarn engineering can reduce the anodic and cathodic resistance and minimize the ohmic loss of the device, increasing the performance (Figure 4e) [4]. Our prior arts demonstrated great potential of the silver (I) oxide with PEDOT:PSS as a stable- and high-preforming cathodic material for flexible biobatteries [5]. To encapsulate the silver-based cathode and promote ion-exchange between the anode and the cathode, a PTFE was selected to form a microporous proton exchange membrane [9]. The cathodic yarn was passivated with the homogenous PTFE membrane at $180^{\circ} \mathrm{C}$ (Figure 4f).

When $S$. oneidensis MR-1 inoculum were introduced to the anodic yarns (Figure 3), the capillary force of the hydrophilic yarns rapidly wicked the anolyte to fill the anodic yarn of the biobattery. While the conductive PEDOT:PSS functionalized anodic yarns harvested electrons from bacterial respiration, the anodic conductive thread provided an efficient electrical pathway through which electrons flowed to the external circuit and reached the cathode. The protons produced by bacterial metabolism
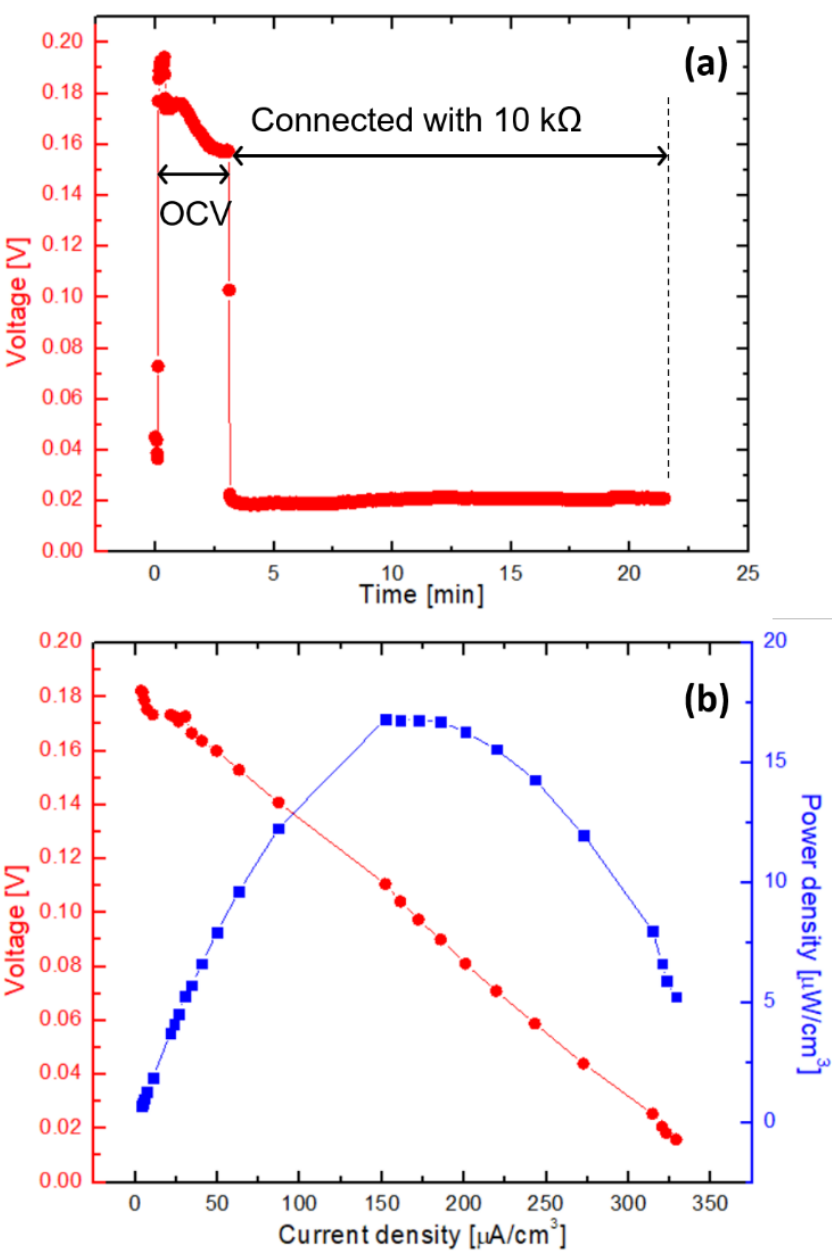

Figure 5: (a) Output voltage measured from a single yarn-based biobattery with or without the external resistor and (b) polarization curveloutput power as a function of current.

traveled to the cathodic yarns through the passivated PTFE membrane. The electrons and protons moved from the anodic yarns finally reduced $\mathrm{Ag}_{2} \mathrm{O}$ to $\mathrm{Ag}$ at the cathodic yarn.

The open circuit voltage $(\mathrm{OCV})$ of $168 \mathrm{mV}$ on average was obtained from the yarn-based biobatteries. A stable current of $2.2 \mu \mathrm{A}$ was produced with a $10 \mathrm{k} \Omega$ resistor between the anode and cathode, providing a proof-of-concept demonstration of the bacterial battery (Figure 5a). The low OCV could be addressed by serially connecting the individual cells with various easy-toconfigure threading or weaving patterns. A cell polarization curve and power output of the battery were measured and obtained based on the saturated current value at a given external resistance (Figure 5b). Slight bending of the textile did not affect the device performance. However, further detailed stretching and bending tests are needed to optimize the device fabrication and configuration. After the device operation, some parts of the PTFE membrane were slightly peeled off from the cathodic yarn (Figure 4f). This exposure of cathode would cause potential releasing of hazardous antibacterial silver ions, cathode biofouling, or shortcircuit between anode and cathode, all of which would negatively affect the biobattery performance. Fabrication improvement of the PTFE membrane would be necessary, and further studies regarding mechanical strength, flexibility, and porosity of the 
membrane are required. Our yarn-based biobattery will be suitable for wearable sensors, such as wrist health monitors [10], textile integrated electrochemical sensors [11], flexible and economical point-of-care diagnostic devices [12].

\section{CONCLUSION}

This work demonstrated the first integration of the bacterialpowered biobattery into yarns with a simple, scalable and fabrication-friendly fashion. The biobattery was built on 1-D yarns through a bottom-up approach, which can be potentially woven into a large energy storage fabric to ultimately deliver high power for practical, flexible applications.

\section{ACKNOWLEDGMENT}

This work is supported by NSF (ECCS \#1503462, IOS \#1543944, \& ECCS \#1703394) and the SUNY Binghamton Research Foundation (SE-TAE).

\section{REFERENCES}

[1] M. Stoppa and A. Chiolerio, "Wearable electronics and smart textiles: A critical review," Sensors, vol. 14, pp. 11957-11992, 2014.

[2] S. Choi, H. Lee, R. Ghaffari, T. Hyeon and D. Kim, "Recent advances in flexible and stretchable Bio-Electronic devices integrated with nanomaterials," Advanced Materials, vol. 28, pp. 4203-4218, 2016.

[3] Q. Huang, D. Wang and Z. Zheng, "Textile-Based electrochemical energy storage devices," Advanced Energy Materials, vol. 6, pp.1600783, 2016.

[4] S. Pang, Y. Gao and S. Choi, "Flexible and stretchable microbial fuel cells with modified conductive and hydrophilic textile," Biosensors and Bioelectronics, vol. 100, pp. 504-511, 2017.

[5] S. Pang, Y. Gao and S. Choi, "Flexible and stretchable biobatteries: Monolithic integration of Membrane-Free microbial fuel cells in a single textile layer," Advanced Energy Materials, vol. 8, pp.1702261, 2017.

[6] Y. Gao and S. Choi, "Stepping toward Self-Powered papertronics: Integrating biobatteries into a single sheet of paper," Advanced Materials Technologies, vol. 2, pp.1600194, 2017.

[7] H. Bi, Z. Yin, X. Cao, X. Xie, C. Tan, X. Huang, B. Chen, F. Chen, $\mathrm{Q}$. Yang and $\mathrm{X}$. Bu, "Carbon fiber aerogel made from raw cotton: A novel, efficient and recyclable sorbent for oils and organic solvents," Advanced Materials, vol. 25, pp. 5916-5921 2013.

[8] C. Kaewprasit, E. Hequet, N. Abidi and J.P. Gourlot, "Quality measurements," J. Cotton Sci, vol. 2, pp. 164-173 1998.

[9] S. Park, J. Lee and B.N. Popov, "Effect of PTFE content in microporous layer on water management in PEM fuel cells," J. Power Sources, vol. 177, pp. 457-463, 2008.

[10] J.M. Nassar, K. Mishra, K. Lau, A.A. Aguirre-Pablo and M.M. Hussain, "Recyclable nonfunctionalized Paper-Based Ultralow-Cost wearable health monitoring system," Advanced Materials Technologies, vol. 2, pp.1600228, 2017.
[11] J. Kim, R. Kumar, A.J. Bandodkar and J. Wang, "Advanced materials for printed wearable electrochemical devices: A review," Advanced Electronic Materials, vol. 3, pp.1600260, 2016.

[12] S. Wang, T. Chinnasamy, M.A. Lifson, F. Inci and U. Demirci, "Flexible substrate-based devices for point-of-care diagnostics," Trends Biotechnol., vol. 34, pp. 909-921, 2016.

\section{CONTACT}

*Seokheun Choi, Assistant Professor, SUNY-Binghamton, Email:sechoi@binghamton.edu Website: http://ws.binghamton.edu/choi 